\title{
IN 17
}

DOI: $10.26461 / 17.10$

\section{Determinación de potenciales contaminantes inorgánicos en muestras de suelos y aguas del territorio uruguayo en la Antártida}

Determination of potential inorganic contaminants in samples of soils and waters of the Uruguayan territory in Antarctica

RECIBIDO: 25/10/2018 $\rightarrow$ APROBADO: 20/11/2018 \mbeluzzi@fq.edu.uy

๑゚ Belluzzi muiños, marcelo (1); lopes fialho, lucimar (2); Pistón, mariela (1)

(1) GRupo de ANÁlisis de ElEMENTOS TRAZA Y DESARROLlo DE ESTRATEGias SIMPLES PARA PREPARACIÓN DE MUESTRAS (GATPREM), ÁREA QUíMICA ANALÍTICA, DEPARTAMENTO ESTRELLA CAMPOS, FACULTAD DE QUÍMICA, UNIVERSIDAD DE LA REPÚBLICA, MONTEVIDEO, URUGUAY

(2) GRUPo DE ANÁlise instrumental APLICADA (GAIA), CENTRO DE CiÊnCIAS EXATAS E DE TECNOLOGIA, DEPARTAMENTO DE QUÍMICA, UNIVERSIDADE FEDERAL DE SÃO CARLOS (UFSCAR), SAN PABLO, BRASIL

\section{RESUMEN}

La investigación científica en la Antártida se ha incrementado en los últimos años, fundamentalmente en áreas como microbiología, biología y oceanografía. Existen pocos reportes sobre los niveles de contaminantes inorgánicos y el impacto antrópico relacionado a ellos en el ecosistema. Al ser un territorio prácticamente virgen, es de particular interés realizar esta evaluación. En este trabajo se analizaron por primera vez y como primera aproximación al abordaje del tema seis muestras de aguas y seis muestras de suelos obtenidas en seis puntos de muestreo próximos a la base uruguaya en Antártida. Los elementos determinados para ambas matrices fueron: cromo, níquel, cobalto, arsénico, estroncio, cadmio, mercurio, plomo, uranio. En agua también se determinaron torio, estaño y antimonio. La preparación de las muestras de suelos se realizó mediante un tratamiento asistido con radiación de microondas; las muestras de agua fueron analizadas directamente. Las determinaciones analíticas se realizaron mediante espectrometría de emisión atómica de plasma de acoplamiento 
inductivo acoplado a masas y óptico (ICP-MS e ICP-OES). La mayoría de los elementos se encuentran dentro de los límites establecidos por algunas agencias de regulaciones ambientales, como Canadian Environmental Quality Guidelines-CCME o United States Environmental Protection Agency-USEPA, y es llamativo fundamentalmente el contenido de estroncio en aguas.

PALABRAS CLAVE: Antártida, contaminantes inorgánicos, impacto antrópico.

\section{ABSTRACT}

Scientific research in Antarctica has increased in recent years, mainly in areas such as Microbiology, Biology and Oceanography. There are few reports about the levels of inorganic contaminants and the anthropic impact related to them in the ecosystem. Being a practically virgin territory, it is of particular interest to carry out this evaluation. In this work, for the first time and as a first approximation to the approach of the subject, six water samples and six soil samples obtained in six sampling points near the Uruguayan base in Antarctica were analyzed. The elements determined for both matrices were: chromium, nickel, cobalt, arsenic, strontium, cadmium, mercury, lead, uranium. In water, thorium, tin and antimony were also determined. The preparation of soil/sediment samples was carried out by means of an assisted treatment with microwave radiation; the water samples were analyzed directly. The analytical determinations were carried out by inductively coupled plasma mass spectrometry (ICP-MS) and optical emission spectrometry (ICP-OES). The majority of the elements are within the limits established by some environmental regulation agencies such as the Canadian Environmental Quality Guidelines-CCME or the United States Environmental Protection Agency-USEPA, being striking strontium content in waters.

KEYWORDS: Antarctica, inorganic contaminants, anthropic impact.

\section{INTRODUCCIÓN}

La Antártida es el continente más austral de la Tierra, posee una superficie de aproximadamente 14 millones de $\mathrm{km}^{2}$, y menos del $1 \%$ se encuentra libre de hielo. Abarca los territorios al sur del paralelo $60^{\circ} \mathrm{S}$, región a la que se refiere el Tratado Antártico firmado el 1 de diciembre de 1959 en Washington (Tratado Antártico, 1959). Presenta un límite natural ubicado entre $\operatorname{los} 55^{\circ}$ y $58^{\circ}$ de latitud sur, denominado Convergencia Antártica, el cual constituye una importante frontera desde el punto de vista biológico y oceanográfico. Este límite está definido por la corriente más intensa del mundo, llamada Corriente Circumpolar Antártica, que rodea al continente en dirección W-E. En promedio es el continente más frío, seco, ventoso y con mayor altura media (más de $2000 \mathrm{~m}$ sobre el nivel del mar) del planeta. A pesar de poseer la mayor reserva de agua dulce del mundo, el continente es considerado un desierto (excepto las zonas costeras e insulares) debido a la 
baja humedad atmosférica, las escasas precipitaciones y, además, al estar el agua en forma de hielo o nieve, no se encuentra disponible para la flora y la fauna. El territorio es prácticamente virgen y es concebido como un continente dedicado a la paz y la ciencia; solamente se registran actividades humanas en las bases científicas y militares que operan allí (Instituto Antártico Uruguayo, 2018).

La investigación científica en el territorio de la Antártida es relevante para el país y aporta al conocimiento no solo a nivel nacional sino también a la comunidad internacional. Existen pocos reportes sobre los niveles de contaminantes inorgánicos y su impacto antrópico en ese ecosistema (De Lima Neto, 2017; Brugnoli, [s.d.]).

Es conocido el impacto sobre la salud humana y ecosistemas de varios metales y semimetales (o especies de ellos) que son elementos clasificados como no esenciales para la vida. En este caso se encuentran varios de los elementos determinados en este trabajo en dos matrices de importancia medioambiental: aguas y suelos (De Azevedo y Da Matta Chasin, 2003).

La Antártida es un continente prácticamente virgen. Es uno de los pocos lugares en el mundo que conservan esa condición, por lo cual es de fundamental importancia la investigación científica en ese territorio. Se presenta un relevamiento primario de los niveles de elementos inorgánicos, algunos de ellos potencialmente tóxicos, como punto de partida para estudios más exhaustivos sobre aguas y suelos en este territorio.

\section{MATERIALES Y MÉTODOS}

\section{Muestras}

Se dispuso de seis muestras de aguas y seis muestras de suelos obtenidos en seis puntos de muestreo próximos a la base uruguaya en Antártida. Las muestras fueron aportadas por la Dra. Silvana Vero del área de Microbiología de la Facultad de Química, quien al momento de los trabajos era responsable de un proyecto de investigación autorizado por el Instituto Antártico Uruguayo. Las muestras de agua fueron colectadas en recipientes de vidrio borosilicato 3.3 con tapa a rosca, transportadas a $1-5{ }^{\circ} \mathrm{C}$ hasta su arribo a los laboratorios de Facultad de Química, acidificadas a $\mathrm{pH}<2$ con $\mathrm{HNO}_{3}$ pa (Asociación Española de Normalización y Certificación, 2003) y posteriormente colocadas en freezer a $-20{ }^{\circ} \mathrm{C}$ hasta el traslado a Brasil, donde nuevamente fueron colocadas en freezer hasta el análisis. Las muestras de suelos fueron colectadas directamente en recipientes de plástico con tapa a rosca y con las mismas condiciones de frío que las muestras de agua hasta que fueron analizadas; en este último caso no se agregaron aditivos. Las coordenadas que ubican los puntos de muestreo se especifican en la Tabla 1. 
TABLA 1. Ubicación geográfica de los puntos de muestreo.

\begin{tabular}{|c|c|c|c|c|}
\hline & \multicolumn{2}{|c|}{ Coordenadas (decimal) } & \multicolumn{2}{|c|}{ Coordenadas (DMS) } \\
\hline $\begin{array}{c}\text { Punto } \\
\text { de muestreo }\end{array}$ & Sur & Oeste & Sur & Oeste \\
\hline Base Artigas & 62.184846 & 58.904239 & $62^{\mathrm{o}} 11^{\prime} 5.4455^{\prime \prime}$ & $58^{\mathrm{O}} 54^{\prime} 15.2603^{\prime \prime}$ \\
\hline Ionosférico & 62.183579 & 58.906307 & $62^{\mathrm{o}} 11^{\prime} 0.8844^{\prime \prime}$ & $58^{\mathrm{o}} 54^{\prime} 22.7051^{\prime \prime}$ \\
\hline Glaciar Collins & 62.184579 & 58.869612 & $62^{\mathrm{o}} 11^{\prime} 4.4843^{\prime \prime}$ & $58^{\circ} 52^{\prime} 10.6031^{\prime \prime}$ \\
\hline Fossil Hill & 62.208494 & 58.970854 & $62^{\mathrm{O}} 12^{\prime} 30.5784^{\prime \prime}$ & $58^{\mathrm{O}} 58^{\prime} 15.0744^{\prime \prime}$ \\
\hline Lago Uruguay & 62.185775 & 58.908140 & $62^{\mathrm{o}} 11^{\prime} 8.7900^{\prime \prime}$ & $58^{\mathrm{o}} 54^{\prime} 29.3040^{\prime \prime}$ \\
\hline $\begin{array}{l}\text { Half Three } \\
\text { Point (HTP) }\end{array}$ & 62.227169 & 58.950031 & $62^{\mathrm{O}} 13^{\prime} 37.8084^{\prime \prime}$ & $58^{\circ} 57^{\prime} 0.1116^{\prime \prime}$ \\
\hline
\end{tabular}

En la Figura 1 se muestran imágenes fotográficas de algunas de las zonas de muestreo y en la Figura 2 se muestran capturas de pantalla de las imágenes satelitales de las seis zonas de muestreo donde se obtuvieron las muestras para este trabajo.

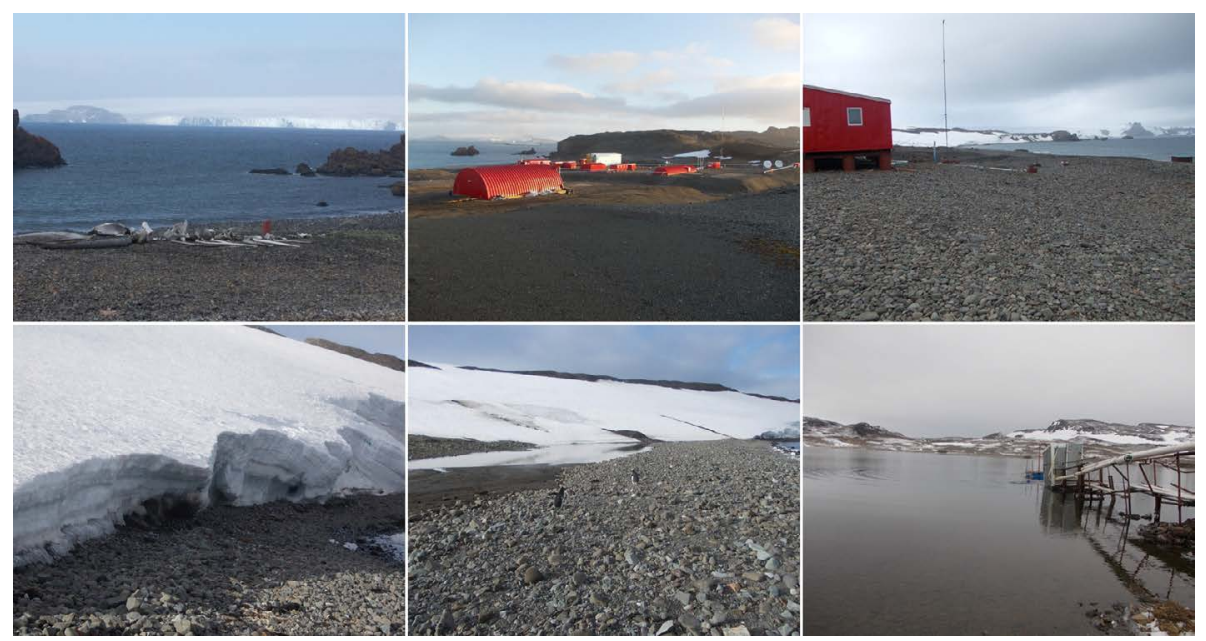

Figura 1. Vista de las zonas de muestreo en la Base Artigas, Glaciar Collins y Lago Uruguay. Fotos cedidas por BC Adalgisa Martínez del Área Microbiología de la Facultad de Química, encargada de los muestreos. 


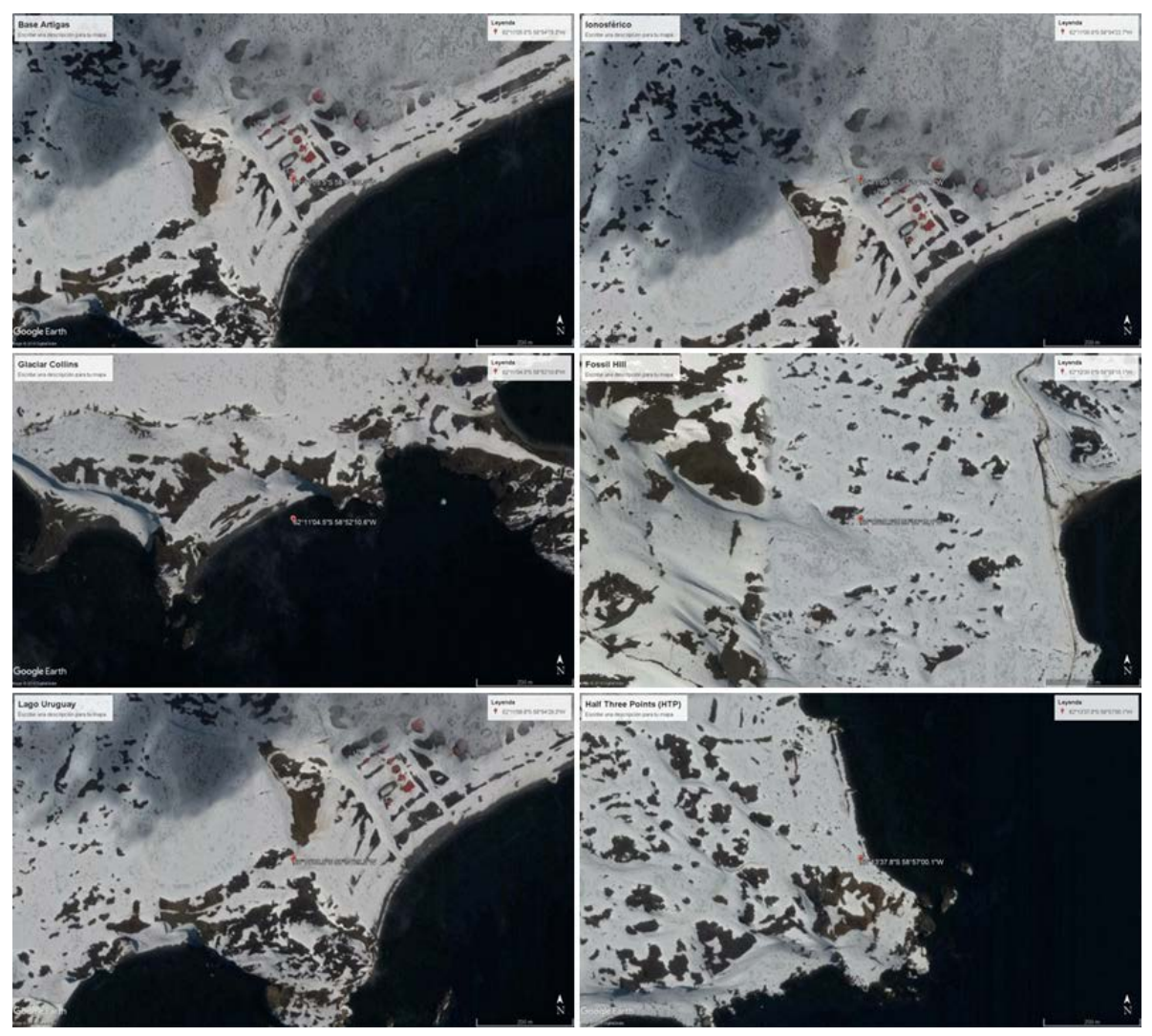

Figura 2. Capturas de pantalla de imágenes satelitales de Google Earth@ de las zonas de muestreo.

\section{Instrumentación}

La digestión de las muestras de suelos fue llevada a cabo mediante un tratamiento asistido con radiación de microondas utilizando un digestor de microondas Ethos 1 (Milestone, Sorisole, Italia).

La determinación de los elementos se realizó mediante espectrometría de emisión atómica de plasma acoplado a masas, habiéndose utilizado un ICP-MS Agilent 7800 (Agilent Technologies, Tokio, JHS, Japón) equipado con nebulizador concéntrico, cámara generadora de aerosol de cuarzo, bomba peristáltica de bajas pulsaciones de 10 cilindros, introducción de muestra mediante sistema HMI (High Matrix Introduction), antorcha de cuarzo con control en tres dimensiones (horizontal, vertical y profundidad), celda de colisión de tipo octapolo (ORS, Octapole Reaction System) y analizador de masas de tipo cuadrupolo. Las mediciones se utilizaron en el modo de colisión He (White Martins-Praxair, Sertãozinho, SP, Brasil) y modo sin gas. Las condiciones de operación se detallan en la Tabla 2. 
TABla 2. Condiciones de operación de plasma ICP-MS.

\begin{tabular}{|cc|}
\hline Parámetro instrumental & Condiciones de operación \\
\hline Potencia RF aplicada $(\mathrm{w})$ & 1550 \\
\hline Flujo de gas de plasma $\left(\mathrm{L} \mathrm{min}^{-1}\right)$ & 12,0 \\
\hline Flujo de gas auxiliar $\left(\mathrm{L} \mathrm{min}^{-1}\right)$ & 1,8 \\
\hline Flujo de gas carrier $\left(\mathrm{L} \mathrm{min}^{-1}\right)$ & 1,0 \\
\hline Flujo de gas He en celda de colisión $\left(\mathrm{mL} \mathrm{min}^{-1}\right)$ & 4,5 \\
\hline Profundidad de introducción de muestra $\left(\mathrm{mm}^{-1}\right)$ & 8 \\
\hline Tiempo de integración $(\mathrm{s})$ & 3,0 \\
\hline Nebulizador & Concéntrico \\
\hline Cámara generadora de aerosol & Scott-doble pase \\
\hline Número de réplicas & 3 \\
\hline
\end{tabular}

La determinación de estroncio se realizó además utilizando un espectrómetro de emisión óptica de plasma acoplado inductivamente (ICP-OES) iCAP6000 (Thermo Fisher Scientific, Waltham, MA, EE.UU.) que funciona en modo de vista radial y axial y está equipado con un detector de CID simultáneo y rejilla Echelle, una bomba peristáltica, nebulizador concéntrico, cámara de pulverización ciclónica y antorcha de plasma desmontable de cuarzo con un inyector de tubo central con un diámetro interno de 2,0 mm. Se seleccionó la línea de emisión de 421,5 nm, que posee una intensidad de emisión adecuada para la determinación. Las condiciones de operación se muestran en la Tabla 3.

TABLA 3. Condiciones de operación de plasma ICP-OES

\begin{tabular}{|cc|}
\hline Parámetro instrumental & Condiciones de operación \\
\hline Potencia RF aplicada $(\mathrm{w})$ & 1150 \\
\hline Flujo de gas de plasma $(\mathrm{L} \mathrm{min}-1)$ & 0,7 \\
\hline Flujo de gas auxiliar $\left(\mathrm{L} \mathrm{min}^{-1}\right)$ & 0,5 \\
\hline Flujo de gas carrier $\left(\mathrm{L} \mathrm{min}^{-1}\right)$ & 12,0 \\
\hline Número de réplicas & 3 \\
\hline
\end{tabular}

Se utilizó argón 99,996\% (White Martins-Praxair, Sertãozinho, SP, Brasil) en ambas espectrometrías. 


\section{Reactivos y patrones}

El material utilizado fue previamente descontaminado utilizando una disolución de $\mathrm{HNO}_{3}$ (Merck pa) $10 \% \mathrm{mv}^{-1}$. Todas las disoluciones fueron preparadas utilizando agua calidad I (Resistividad no menor a 18,2 M $\Omega \mathrm{cm}$ ) según especificaciones de la American Society for Testing and Materials (American Society for Testing and Materials, 2006) obtenida de un sistema de purificación de agua Milli-Q@ Plus Total Water System (Millipore Corp., Bedford, MA, USA). Los patrones de calibración se prepararon a partir de disoluciones $1000 \mathrm{mg} \mathrm{L}^{-1}$ (Qhemis, São Paulo,SP, Brazil). Para la digestión de las muestras se utilizó $\mathrm{HNO}_{3}$ (Merck pa) previamente destilado utilizando un sistema de destilación sub-boiling distillation system (Milestone, Sorisole, Italia) y $\mathrm{H}_{2} \mathrm{O}_{2} 30 \% \mathrm{mv}^{-1}$ (Labsynth, Diadema, Brasil).

\section{Preparación de muestras}

Las muestras de suelos fueron tratadas en mortero de ágata para reducir el tamaño de partícula y homogeneizar. Se tomaron masas de muestra de aproximadamente $200 \mathrm{mg}$ y se agregó 5,0 mL de $\mathrm{HNO}_{3} 7 \mathrm{~mol} \mathrm{~L}-1$ y $3,0 \mathrm{~mL}$ de $\mathrm{H}_{2} \mathrm{O}_{2} 30 \% \mathrm{mv}^{-1}$ para la digestión asistida con radiación de microondas (Krug, 2016). El programa de calentamiento utlizado fue: (1) 5 min para alcanzar $160{ }^{\circ} \mathrm{C}$; (2) mantenimiento de temperatura $\left(160{ }^{\circ} \mathrm{C}\right.$ ) durante $5 \mathrm{~min}$; (3) $10 \mathrm{~min}$ para alcanzar $240{ }^{\circ} \mathrm{C}$; (4) mantenimiento de temperatura $\left(240{ }^{\circ} \mathrm{C}\right)$ durante $10 \mathrm{~min}$; (5) enfriamiento del sistema. Cada muestra de suelo y los blancos fueron preparados por duplicado.

Las muestras de aguas fueron medidas directamente sin tratamiento alguno.

\section{Determinaciones analíticas}

Los elementos determinados para ambas matrices fueron: cromo, níquel, cobalto, arsénico, estroncio, cadmio, mercurio, plomo, uranio; en agua además se determinaron los elementos torio, estaño y antimonio. Se analizaron los isótopos más abundantes de cada elemento (Perkin Elmer SCIEX, 2008).

TABLA 4. Elementos determinados, isótopos analizados y su abundancia. Información obtenida de Perkin Elmer SCIEX (2008).

\begin{tabular}{|cccc}
\hline Elemento & Símbolo & Isótopo & Abundancia (\%) \\
Cromo & $\mathrm{Cr}$ & ${ }^{52} \mathrm{Cr}$ & 83,79 \\
& & ${ }^{53} \mathrm{Cr}$ & 9,50 \\
\hline Níquel & $\mathrm{Ni}$ & ${ }^{58} \mathrm{Ni}$ & 68,08 \\
\hline Cobalto & ${ }^{60} \mathrm{Ni}$ & 26,22 \\
\hline Arsénico & $\mathrm{Co}$ & ${ }^{59} \mathrm{Co}$ & 100,00 \\
\hline
\end{tabular}




\begin{tabular}{|c|c|c|c|}
\hline Elemento & Símbolo & Isótopo & Abundancia (\%) \\
\hline \multirow{2}{*}{ Estroncio } & \multirow{2}{*}{$\mathrm{Sr}$} & ${ }^{86} \mathrm{Sr}$ & 9,86 \\
\hline & & ${ }^{88} \mathrm{Sr}$ & 82,58 \\
\hline \multirow{2}{*}{ Cadmio } & \multirow{2}{*}{$\mathrm{Cd}$} & ${ }^{112} \mathrm{Cd}$ & 24,13 \\
\hline & & ${ }^{114} \mathrm{Cd}$ & 28,73 \\
\hline \multirow{2}{*}{ Estaño } & \multirow{2}{*}{$\mathrm{Sn}$} & ${ }^{118} \mathrm{Sn}$ & 24,23 \\
\hline & & ${ }^{120} \mathrm{Sn}$ & 32,59 \\
\hline \multirow{2}{*}{ Antimonio } & \multirow{2}{*}{$\mathrm{Sb}$} & ${ }^{121} \mathrm{Sb}$ & 57,36 \\
\hline & & ${ }^{123} \mathrm{Sb}$ & 42,64 \\
\hline \multirow{2}{*}{ Mercurio } & \multirow{2}{*}{$\mathrm{Hg}$} & ${ }^{200} \mathrm{Hg}$ & 23,10 \\
\hline & & ${ }^{202} \mathrm{Hg}$ & 29,86 \\
\hline \multirow{3}{*}{ Plomo } & \multirow{3}{*}{$\mathrm{Pb}$} & ${ }^{206} \mathrm{~Pb}$ & 24,10 \\
\hline & & ${ }^{207} \mathrm{~Pb}$ & 22,10 \\
\hline & & ${ }^{208} \mathrm{~Pb}$ & 52,40 \\
\hline Torio & Th & ${ }^{232} \mathrm{Th}$ & 100,00 \\
\hline \multirow{2}{*}{ Uranio } & \multirow{2}{*}{$\mathrm{U}$} & ${ }^{235} \mathrm{U}$ & 0,72 \\
\hline & & ${ }^{238} \mathrm{U}$ & 99,27 \\
\hline
\end{tabular}

\section{Calibración}

Para el análisis mediante ICP-MS se realizó para todos los elementos curva de calibración con patrones de concentración 0,$00 ; 0,01 ; 0,05 ; 0,10 ; 0,50 ; 1,00 ; 5,00$; 10,00 y $50,00 \mu \mathrm{g} \mathrm{L}^{-1}$, mediante diluciones apropiadas a partir de las disoluciones stock de aproximadamente $1000 \mathrm{mg} \mathrm{L}^{-1}$ de cada elemento. Se utilizó un método de calibración de compatibilización matricial entre disoluciones de calibración y muestras (matrix match).

La calibración para el análisis de estroncio mediante ICP-OES fue realizada con patrones de concentración 0,$00 ; 0,50 ; 1,00 ; 1,50 ; 3,00 ; 5,00$ y 10,00 $\mathrm{mg} \mathrm{L}^{-1}$, preparados mediante diluciones apropiadas a partir de la disolución stock correspondiente.

Para las determinaciones mediante ICP-MS se estudiaron los siguientes parámetros de desempeño: linealidad, límite de detección, límite de cuantificación y veracidad. Para realizar la evaluación de veracidad de todos los elementos determinados excepto estroncio se fortificaron muestras a dos niveles de concentración (adición de $1 \mu \mathrm{g} \mathrm{L}^{-1}$ y $2 \mu \mathrm{g} \mathrm{L}^{-1}$ de patrones de cada analito). Para el estudio de veracidad en la determinación de estroncio, las muestras diluidas 100 veces se adicionaron con 10 y $25 \mu \mathrm{g} \mathrm{L}^{-1}$ de un patrón de $\mathrm{Sr}$.

Las determinaciones mediante ICP-OES en las muestras de agua se realizaron para confirmar los resultados obtenidos para el estroncio mediante ICP-MS; en este caso se evaluó linealidad y veracidad. Para el estudio de veracidad, las muestras se diluyeron en proporción $1: 1 ; 1: 2 ; 1: 3$ y $1: 4$. 


\section{RESULTADOS}

\section{Resultados de la validación}

En las Tablas 5, 6 y 7 se presentan los resultados obtenidos de los parámetros de desempeño evaluados y en las Tablas 8, 9 y 10 los resultados obtenidos en las muestras analizadas.

TABLA 5. Resultado de algunos parámetros de validación mediante ICP-MS.

\begin{tabular}{ccc}
\hline Parámetro & Concepto/unidad & Resultado \\
Linealidad & Ajuste a modelo lineal & Cumple \\
& $\mathrm{R}^{2}$ & $>0,9996$ \\
\hline & $\% \mathrm{R}$ (agua) & $72,0-107,5$ \\
Veracidad & $\% \mathrm{R}$ (suelos) & Promedio 90,9 \\
& & $71,0-132,5$ \\
& & Promedio 95,3
\end{tabular}

\%R: porcentaje de recuperación.

TABLA 6. Resultados de límites de detección (LD) y límites de cuantificación (LC) mediante ICP-MS.

\begin{tabular}{|c|c|c|}
\hline Elemento & $\mathrm{LD}\left(\mu \mathrm{g} \mathrm{L}^{-1}\right)$ & $\mathrm{LC}\left(\mu \mathrm{g} \mathrm{L}^{-1}\right)$ \\
\hline $\mathrm{Cr}$ & 0,017 & 0,057 \\
\hline $\mathrm{Ni}$ & 0,014 & 0,047 \\
\hline $\mathrm{Co}$ & 0,005 & 0,017 \\
\hline As & 0,014 & 0,047 \\
\hline $\mathrm{Sr}$ & 0,040 & 0,133 \\
\hline $\mathrm{Cd}$ & 0,001 & 0,004 \\
\hline $\mathrm{Sn}$ & 0,015 & 0,050 \\
\hline $\mathrm{Sb}$ & 0,003 & 0,011 \\
\hline $\mathrm{Hg}$ & 0,013 & 0,043 \\
\hline $\mathrm{Pb}$ & 0,010 & 0,033 \\
\hline Th & 0,001 & 0,002 \\
\hline $\mathrm{U}$ & 0,001 & 0,002 \\
\hline Criterio & $" 3 s "$ & "10s" \\
\hline
\end{tabular}


TABLA 7. Resultados de los parámetros validados por ICP-OES.

\begin{tabular}{|ccc}
\hline Parámetro & Concepto/unidad & Resultado \\
\hline Linealidad & Ajuste a modelo lineal & Cumple \\
\hline Veracidad & $\mathrm{R}^{2}$ & 0,9984 \\
\hline
\end{tabular}

\section{Resultados en muestras de agua}

TABLA 8. Resultados en las muestras de aguas mediante ICP-MS (ND: no detectado).

\begin{tabular}{|c|c|c|c|}
\hline Muestra & $\mathrm{Cr}$ & $\mathbf{N i}$ & Co \\
\hline Base Artigas & $0,641 \pm 0,046$ & $1,467 \pm 0,052$ & $0,062 \pm 0,055$ \\
\hline Half Three Points (нтP) & $0,473 \pm 0,071$ & $0,113 \pm 0,011$ & $0,00914 \pm 0,00090$ \\
\hline Fossil Hill & $0,473 \pm 0,050$ & $0,1128 \pm 0,0045$ & $0,0065 \pm 0,0035$ \\
\hline Ionosférico & $0,422 \pm 0,052$ & $0,1038 \pm 0,0084$ & $0,0071 \pm 0,0010$ \\
\hline Glaciar Collins & $0,429 \pm 0,024$ & $0,1034 \pm 0,0024$ & $0,0087 \pm 0,0045$ \\
\hline Lago Uruguay & $0,522 \pm 0,053$ & $0,1881 \pm 0,0084$ & $0,00601 \pm 0,00090$ \\
\hline Muestra & As & $\mathbf{S r}$ & Cd \\
\hline Base Artigas & $0,965 \pm 0,064$ & $3809 \pm 12$ & $0,099 \pm 0,039$ \\
\hline Half Three Points (нтР) & $0,956 \pm 0,012$ & $3971,9 \pm 2,2$ & $0,0216 \pm 0,0012$ \\
\hline Fossil Hill & $0,854 \pm 0,034$ & $3611 \pm 18$ & $0,0280 \pm 0,0023$ \\
\hline Ionosférico & $0,822 \pm 0,074$ & $3412,0 \pm 9,0$ & $0,0199 \pm 0,0067$ \\
\hline Glaciar Collins & $0,808 \pm 0,018$ & $3288 \pm 16$ & $0,0192 \pm 0,0022$ \\
\hline Lago Uruguay & $0,810 \pm 0,021$ & $3422 \pm 20$ & $0,0213 \pm 0,0045$ \\
\hline Muestra & $\mathrm{Hg}$ & $\mathbf{P b}$ & $\mathbf{U}$ \\
\hline Base Artigas & $0,233 \pm 0,034$ & $0,0206 \pm 0,0039$ & $0,769 \pm 0,046$ \\
\hline Half Three Points (HTP) & $0,1129 \pm 0,0082$ & ND & $0,6971 \pm 0,0051$ \\
\hline Fossil Hill & $0,134 \pm 0,016$ & ND & $0,5829 \pm 0,0083$ \\
\hline Ionosférico & $0,0528 \pm 0,0039$ & ND & $0,5291 \pm 0,0032$ \\
\hline Glaciar Collins & $0,0917 \pm 0,0067$ & ND & $0,5397 \pm 0,0057$ \\
\hline Lago Uruguay & $0,0213 \pm 0,0041$ & ND & $0,572 \pm 0,016$ \\
\hline
\end{tabular}




\begin{tabular}{|cccc|}
\hline Muestra & Th & Sn & Sb \\
\hline Base Artigas & $0,0250 \pm 0,0035$ & $0,0357 \pm 0,0053$ & $0,0895 \pm 0,0025$ \\
\hline Half Three Points (HTP) & ND & ND & $0,0706 \pm 0,0043$ \\
\hline Fossil Hill & $<0,002$ & ND & $0,0619 \pm 0,0028$ \\
\hline Ionosférico & ND & ND & $0,0582 \pm 0,0014$ \\
\hline Glaciar Collins & $0,0027 \pm 0,0023$ & ND & $0,0539 \pm 0,0078$ \\
\hline Lago Uruguay & ND & ND & $0,0596 \pm 0,0036$ \\
\hline Resultados \pm desviación estándar $\left(\boldsymbol{\mu g} \mathbf{L}^{-1}\right)$ & \\
\hline
\end{tabular}

\section{Resultados en muestras de suelos}

TABLA 9. Resultados de las muestras de suelos mediante ICP-MS.

En los casos en que el criterio adoptado para la estimación de la incertidumbre arrojara resultados no coherentes (muestras con muy bajo contenido del analito), solamente se expresó el resultado de la cuantificación. ND: no detectado.

\begin{tabular}{|cccc|}
\hline Muestra & Cr & Ni & Co \\
\hline Base Artigas & $2,7 \pm 1,7$ & $2,7 \pm 1,9$ & $12,5 \pm 0,1$ \\
\hline Half Three Points (HTP) & $17,5 \pm 0,3$ & $9,7 \pm 0,5$ & $17,0 \pm 0,2$ \\
\hline Fossil Hill & $15,2 \pm 0,1$ & $6,1 \pm 0,2$ & $20,7 \pm 0,2$ \\
\hline Ionosférico & $22,3 \pm 0,3$ & $8,7 \pm 0,2$ & $13,2 \pm 0,5$ \\
\hline Glaciar Collins & $2,3 \pm 2,0$ & $2,1 \pm 1,5$ & $8,1 \pm 1,4$ \\
\hline Lago Uruguay & $4,6 \pm 2,5$ & $2,6 \pm 1,8$ & $11,9 \pm 0,6$ \\
\hline Muestra & $\mathbf{A s}$ & $\mathbf{S r}$ & $\mathbf{C d}$ \\
\hline Base Artigas & $1,3 \pm 1,0$ & $391,5 \pm 0,5$ & 0,08 \\
\hline Half Three Points (HTP) & $0,4 \pm 0,3$ & $403,1 \pm 0,1$ & 0,04 \\
\hline Fossil Hill & $1,8 \pm 0,7$ & $161,7 \pm 0,1$ & 0,08 \\
\hline Ionosférico & $13,2 \pm 0,6$ & $288,4 \pm 0,1$ & 0,11 \\
\hline Glaciar Collins & $8,1 \pm 1,5$ & $237,6 \pm 0,1$ & 0,04 \\
\hline Lago Uruguay & $11,9 \pm 0,7$ & $322,1 \pm 0,1$ & 0,10 \\
\hline
\end{tabular}




\begin{tabular}{|cccc}
\hline Muestra & Hg & Pb & U \\
\hline Base Artigas & 0,06 & $5,8 \pm 0,5$ & $1,0 \pm 0,8$ \\
\hline Half Three Points (HTP) & ND & $1,8 \pm 1,1$ & $0,6 \pm 0,5$ \\
\hline Fossil Hill & 0,52 & $2,6 \pm 0,8$ & 0,47 \\
\hline Ionosférico & ND & $4,4 \pm 0,8$ & 0,57 \\
\hline Glaciar Collins & ND & $2,5 \pm 0,7$ & 0,67 \\
\hline Lago Uruguay & ND & $3,6 \pm 1,5$ & $1,2 \pm 0,6$ \\
\hline Resultados $\left(\mathbf{m g} \mathbf{~ k g}^{-1}\right.$ ) \pm (diferencia porcentual entre réplicas $/$ promedio)
\end{tabular}

TABLA 10. Resultados del contenido de estroncio mediante ICP-OES en aguas.

\begin{tabular}{|cc|}
\hline Muestra & Sr $\left(\mathbf{m g ~ \mathbf { L } ^ { - 1 }}\right)$ \\
\hline Base Artigas & $4,076 \pm 0,010$ \\
\hline Half Three Points (HTP) & $4,1576 \pm 0,0053$ \\
\hline Fossil Hill & $3,9934 \pm 0,0068$ \\
\hline Ionosférico & $3,8545 \pm 0,0078$ \\
\hline Glaciar Collins & $3,777 \pm 0,024$ \\
\hline Lago Uruguay & $4,141 \pm 0,027$ \\
\hline Resultados \pm desviación estándar $\left(\mathbf{m g ~ \mathbf { L } ^ { - 1 }}\right)$ \\
\hline
\end{tabular}

\section{DISCUSIÓN}

La validación de la metodología para las dos técnicas instrumentales utilizadas arrojó resultados satisfactorios para el fin propuesto, adecuados para estudios ambientales.

\section{Resultados en muestras de aguas}

Algunas agencias de regulaciones ambientales, como Canadian Environmental Quality Guidelines - CCME o United States Environmental Protection Agency - US EPA (United States Environmental Protection Agency - USEPA, [s.d.]), establecen límites máximos para contaminantes inorgánicos en aguas. CCME publica una guía que refiere a aguas de consumo y aguas marinas (Canadian Council of Ministers of the Environment - CCME, 2007a, 2007b). US EPA también publica guías de referencia y recomendaciones para aguas de consumo (Safe Drinking Water Act y 2018 Drinking Water Standards and Health Advisories Tables) que establecen el contenido máximo admisible de contaminantes inorgánicos en agua de consumo humano. Si bien las aguas estudiadas no son de consumo directo, igualmente se 
toman en cuenta las recomendaciones de las agencias antes citadas para ese tipo de aguas. Un resumen de los contenidos máximos sugeridos se presenta en la Tabla 11.

TABLA 11. Contenidos máximos admisibles recomendados por CCME y US EPA para aguas de consumo. N/D: no declarado.

\begin{tabular}{|c|c|c|c|c|c|c|c|}
\hline Agencia & Unidades & $\mathrm{Cr}$ & $\mathbf{N i}$ & Co & As & $\mathrm{Sr}$ & Cd \\
\hline CCME & $\mu \mathrm{g} \mathrm{L}^{-1}$ & 1,5 & $\mathrm{~N} / \mathrm{D}$ & $\mathrm{N} / \mathrm{D}$ & 12,5 & $\mathrm{~N} / \mathrm{D}$ & 0,12 \\
\hline US EPA & $\mathrm{mg} \mathrm{L}^{-1}$ & 0,1 & $\mathrm{~N} / \mathrm{D}$ & $\mathrm{N} / \mathrm{D}$ & 0,01 & 4 & 0,005 \\
\hline Agencia & Unidades & $\mathbf{H g}$ & $\mathbf{P b}$ & $\mathbf{U}$ & $\mathbf{T h}$ & Sn & $\mathrm{Sb}$ \\
\hline CCME & $\mu g \mathrm{~L}^{-1}$ & 0,016 & $\mathrm{~N} / \mathrm{D}$ & $\mathrm{N} / \mathrm{D}$ & $\mathrm{N} / \mathrm{D}$ & $\mathrm{N} / \mathrm{D}$ & $\mathrm{N} / \mathrm{D}$ \\
\hline US EPA & $\mathrm{mg} \mathrm{L}^{-1}$ & 0,002 & 0,015 & $\mathrm{~N} / \mathrm{D}$ & $\mathrm{N} / \mathrm{D}$ & $\mathrm{N} / \mathrm{D}$ & 0,006 \\
\hline
\end{tabular}

Los contenidos de mercurio en las seis muestras analizadas cumplen con los límites establecidos por US EPA pero superan los límites establecidos por CCME. Respecto al contenido de cadmio, una de las muestras presenta un contenido del metal que se encuentra cerca del límite del valor máximo sugerido por CCME pero cumple con lo establecido por US EPA en todas las muestras analizadas. Particularmente es de destacar el contenido de estroncio en las muestras de agua. La US EPA ha establecido un límite de 4,0 $\mathrm{mg} \mathrm{L}^{-1}$ para este metal en agua de consumo directo (2018 Drinking Water Standards and Health Advisories Tables). Los contenidos de estroncio determinados por ICP-OES en las seis muestras están en el entorno del valor máximo sugerido por esa agencia, tres de ellas poco por debajo y tres de ellas por encima de ese valor (resultados en el rango 3,78 - 4,16 $\mathrm{mg} \mathrm{L}^{-1}$ ). El valor promedio obtenido en los puntos muestreados fue precisamente el del valor máximo sugerido por US EPA. Estos resultados son del mismo orden que los determinados por ICP-MS (resultados en el rango 3,29-3,97 mg L-1). Si bien son pocas muestras, es un estudio de screening para obtener los primeros datos sobre niveles de estroncio, pues no se conocen antecedentes sobre estudios de este tipo.

\section{Resultados en muestras de suelos}

Para esta discusión se toman en cuenta los valores máximos recomendados por CCME para suelos de parques. En la Tabla 12 se resumen estos valores.

TABLA 12. Valores máximos recomendados de CCME de contaminantes inorgánicos en suelos de parques. N/D: no declarado.

\begin{tabular}{|ccccccc|}
\hline Agencia & Unidades & $\mathbf{C r}$ & $\mathbf{N i}$ & $\mathbf{C o}$ & $\mathbf{A s}$ & $\mathbf{S r}$ \\
CCME & $\mathrm{mg} \mathrm{kg}^{-1}$ & 64 & 50 & $\mathrm{~N} / \mathrm{D}$ & 12 & $\mathrm{~N} / \mathrm{D}$ \\
\hline Agencia & Unidades & $\mathbf{C d}$ & $\mathbf{H g}$ & $\mathbf{P b}$ & $\mathbf{U}$ \\
CCME & $\mathrm{mg} \mathrm{kg}^{-1}$ & 10 & 6,6 & 140 & 23
\end{tabular}


La observación de los resultados obtenidos permite visualizar que en general las muestras analizadas cumplen con las recomendaciones de la Canadian Environmental Quality Guidelines (Canadian Environmental Quality Guidelines, 2007). Si bien no está declarado el contenido máximo de estroncio en las recomendaciones que publica esa agencia, los resultados obtenidos podrían indicar que el contenido de este metal puede resultar alto. El estroncio está asociado a rocas, es un elemento relativamente abundante en la naturaleza y representa una media del 0,034\% de todas las rocas ígneas. Se encuentra mayoritariamente en forma de sulfato en el mineral celestina y carbonato en el mineral estroncianita (U.S. Geological Survey, 2010).

\section{CONCLUSIONES}

Los estudios presentados en este trabajo están entre los primeros dirigidos a relevar el impacto antropogénico en el territorio antártico a partir del monitoreo de los niveles de concentración de los elementos indicados, y es un punto de partida de futuros proyectos de monitoreo ambiental. Particularmente debería ponerse especial atención a los contenidos de cadmio, mercurio y estroncio en aguas y estroncio en suelos. En relación a este último metal, sería interesante determinar cuál es el isótopo predominante para evaluar posible actividad nuclear en la zona (Agencia para Sustancias Tóxicas y el Registro de Enfermedades, [s.d.]; Baeza, 1994). Si bien en este trabajo los contenidos de estaño, plomo y antimonio no resultaron relevantes, igualmente son interesantes de monitorear porque pueden ser indicadores de contaminación por residuos de cascos de los barcos.

Asimismo, resulta de importancia la ampliación de los estudios a otras matrices, como por ejemplo algas (Farías, et al., 2002; Farías, et al., 2007), especies marinas y otras especies comunes en la zona.

\section{RECONOCIMIENTOS}

Se agradece al Instituto Antártico Uruguayo por autorizar la investigación científica y publicación de este trabajo y al Dr. Álvaro Soutullo como jerarca responsable de las actividades en ese territorio. A la Dra. Silvana Vero y Dra. Silvana Alborés del Área Microbiología de la Facultad de Química - UdelaR, responsables ante el I.A.U. de los muestreos y quienes aportaron gentilmente las muestras utilizadas en este trabajo. A los colegas que participaron de los muestreos y que aportaron información relevante para la confección de este texto.

A PEDECIBA Química.

Al profesor Joaquim de Araújo Nóbrega y a los colegas del laboratorio GAIA, quienes brindaron su apoyo y permanentemente disposición para la realización de este trabajo. A través de ellos, agradecemos a la Universidade Federal de São Carlos (UFSCar), en cuyas instalaciones y laboratorios fueron desarrollados los ensayos.

A Agilent Technologies, Thermo Scientific and Analítica. 


\section{REFERENCIAS}

Agencia para Sustancias Tóxicas y el Registro de Enfermedades, [s.d.]. Resúmenes de Salud Pública - Estroncio (Strontium) [En línea]. Atlanta: ATSDR. [Consulta: 15 de octubre de 2018]. Disponible en: https://www.atsdr.cdc.gov/es/phs/es_phs159.html

American Society for Testing and Materials, 2006. ASTM 1193: Standard specification for reagent water. Philadelphia: ASTM.

Asociación Española de Normalización y Certificación, 2003. UNE-EN 5667-2: Guía para la conservación y manipulación de las muestras de agua. Madrid: AENOR

Baeza , A., Del Río, M., Paniagua, J., Navarro , E., Rodriguez, M. y Sánchez, F., 1994. Radioactive concentrations of the livingston island soils (Antarctica). Dosimetry considerations. En: Appl. Radiat. Isot., 6, pp.675-681.

Brugnoli, E., Colina, R., Victoria, M., Venturini, N., Bueno, C., Iglesias, K. y otros, [s.d]. Identificación de los impactos antrópicos en ambientes cercanos a la Base Científica Antártica Artigas (Isla Rey Jorge), mediante el uso de indicadores químicos y microbiológicos (02/2014 - 03/2016). Proyecto de investigación con apoyo financiero del Instituto Antártico Uruguayo. Inédito

Canadian Council of Ministers of the Environment - CCME, 2007a. Canadian environmental quality guidelines [En línea]. Winnipeg: CCME. [Consulta: 15 de octubre de 2018]. Disponible en: https://www.ccme.ca/en/resources/canadian_ environmental_quality_guidelines/

Canadian Council of Ministers of the Environment - CCME, 2007b. Canadian soil quality guidelines for the protection of environmental and human health [En línea]. Winnipeg: CCME. [Consulta: 15 de octubre de 2018]. Disponible en: http://esdat. net/Environmental\%20Standards/Canada/SOIL/rev_soil_summary_tbl_7.0_e.pdf

De Azevedo, F. A. y Da Matta Chasin, A., 2003. Metais. Gerenciamiento da toxicidade. San Pablo: Atheneu, InterTox

De Lima Neto, E., Braga Bueno Guerra, M., Thomazini, A., Daher, M., Medeiros de Andrade, A. y Schaefer G.R., C. E., 2017. Soil contamination by toxicmetals near an Antarctic Refuge in Robert Island,Maritime Antarctica: a monitoring strategy. En: Water Air Soil Pollut, 228, pp.228-66.

Farías, S., Pérez Arisnabarreta, S., Vodopidez, C. y Smichowsky, P., 2002. Levels of essential and potentially toxic trace metals in Antarctic macro algae. En: Spectrochimica Acta Part B, 57, pp.2133-2140.

Farías, S., Smichowsky, P., Vélez, D., Montoro, R., Curtosi, A. y Vodopívez, C., 2007. Total and inorganic arsenic in Antarctic macroalgae. En: Chemosphere, 69, pp.1017-1024.

Instituto Antártico Uruguayo, 2018. Publicaciones científicas 1997-2018 [En línea]. Montevideo: Instituto Antártico Uruguayo. [Consulta: 12 de octubre de 2018]. Disponible en: http://www.iau.gub.uy/?page_id=1953\&lang=es

Krug, F. y Piovezani Rocha, F. R., 2016. Métodos de preparo de amostras para análise elementar. San Pablo: Sociedade Brasileira de Química. pp.315-383.

Perkin Elmer SCIEX, 2008. Isotopo abundances, interferences and DRC information. Massachusetts: Perkin. 
Tratado Antártico, 1959 [En línea]. Buenos Aires: Secretaría del Tratado Antártico. [Consulta: 11 de octubre de 2018]. Disponible en: http://www.ats.aq/documents/ keydocs/vol_1/vol1_2_AT_Antarctic_Treaty_s.pdf

U.S. Environmental Protection Agency, 2018. 2018 Edition of the drinking water standards and health advisories tables [En línea]. Washington: USEPA. [Consulta: 20 de octubre de 2018]. Disponible en: de https://www.epa.gov/sites/production/ files/2018-03/documents/dwtable2018.pdf

U.S. Geological Survey, 2010. 2007 minerals yearbook [En línea]. Virginia: USGS. [Consulta: 15 de octubre de 2018]. Disponible en: https://minerals.usgs.gov/ minerals/pubs/commodity/strontium/myb1-2007-stron.pdf

United States Environmental Protection Agency - USEPA, [s.d.]. Safe drinking water act (SDWA) [En línea]. [s.l.]: USEPA [Consulta: 15 de octubre de 2018]. Disponible en: https://www.epa.gov/sdwa

\section{Páginas web recomendadas}

Instituto Antártico Uruguayo: http://www.iau.gub.uy/?lang=es 